\title{
Effect of the Dipeptidyl Peptidase-4 Inhibitor Sitagliptin on Muscle Mass and the Muscle/Fat Ratio in Patients With Type 2 Diabetes
}

\author{
Satoshi Ishii ${ }^{a}$, Yoshio Nagaia, ${ }^{a}$, Hiroyuki Kato ${ }^{a}$, Hisashi Fukuda ${ }^{a}$, Yasushi Tanaka ${ }^{a}$
}

\begin{abstract}
Background: We previously reported the effect of sitagliptin or glimepiride treatment for 24 weeks on body composition in Japanese overweight and obese patients with type 2 diabetes. Although the degree of $\mathrm{HbAlc}$ reduction was similar between the two groups, significant reduction of intrahepatic lipid (IHL), determined by proton magnetic resonance spectroscopy $\left({ }^{1} \mathrm{H}-\mathrm{MRCP}\right)$, and fat mass (FM), determined by dual-energy X-ray absorptiometry (DXA), was observed in the sitagliptin group but not in the glimepiride group. As both IHL and FM are known as associating factors of insulin resistance, these reductions may lead to improvement of insulin sensitivity, which in turn may contribute to sitagliptin-induced amelioration of glycemic control. On the other hand, muscle and muscle/fat ratio were also reported to be positively correlated with insulin sensitivity, but we did not evaluate these factors.
\end{abstract}

Methods: DXA separates the whole body into three major components, bone mass (BM), FM and fat and bone-free mass (FBFM), and measures the weight of each component. FBFM is normally used as a good marker of muscle mass; therefore, in this post-hoc analysis, we investigated whether sitagliptin treatment for 24 weeks influenced the FBFM and FBFM/FM ratio.

Results: After 24 weeks, the FBFM and FBFM/FM ratio significantly increased in the sitagliptin group $(47.6 \pm 10.3$ to $48.8 \pm 11.0 \mathrm{~kg}, \mathrm{P}<$ 0.05 and $2.0 \pm 0.8$ to $2.1 \pm 0.8, \mathrm{P}<0.05)$, but not in the glimepiride group $(49.7 \pm 10.6$ to $49.3 \pm 9.9, \mathrm{P}=0.655$ and $2.1 \pm 0.9$ to $2.0 \pm 0.7$, $\mathrm{P}=0.855$ ). The mean change of FBFM and FBFM/FM ratio from baseline to 24 weeks in the sitagliptin and glimepiride groups was $1.24 \pm 2.01$ (sitagliptin group) vs. $-0.34 \pm 2.63 \mathrm{~kg}$ (glimepiride group) $(\mathrm{P}=0.074)$ and $0.13 \pm 0.17$ (sitagliptin group) vs. $-0.11 \pm 0.30$ (glimepiride group) $(\mathrm{P}<0.05)$, respectively.

Manuscript submitted December 27, 2019, accepted January 17, 2020

aDivision of Metabolism and Endocrinology, Department of Internal Medicine, St. Marianna University School of Medicine, Kawasaki, Japan

${ }^{\mathrm{b}}$ Corresponding Author: Yoshio Nagai, Division of Metabolism and Endocrinology, Department of Internal Medicine, St. Marianna University School of Medicine, 2-16-1, Sugao, Miyamae-ku, Kawasaki, Kanagawa 216-8511, Japan. Email: ynagai@marianna-u.ac.jp

doi: https://doi.org/10.14740/jocmr4078
Conclusions: Sitagliptin 24-week treatment demonstrated not only reduction of body fat and liver fat but also an increase of muscle and muscle/fat ratio. These changes may partly explain the mechanism underlining sitagliptin-induced improvement of glycemic control.

Keywords: Sitagliptin; Muscle mass; Muscle/fat ratio; Type 2 diabetes mellitus

\section{Introduction}

We previously reported that treatment with the sodium glucose co-transporter 2 (SGLT2) inhibitor, ipragliflozin, for 24 weeks reduced not only body fat and intrahepatic lipid (IHL) but also muscle mass in the patients with type 2 diabetes [1]. Moreover, we recently revealed that 24 weeks of treatment with the glucagon-like peptide-1 (GLP-1) receptor agonist, liraglutide, decreased body fat and IHL to a similar degree, but did not reduce the muscle in patients with type 2 diabetes [2]. Rather, in animal models, the GLP-1 receptor agonist and dipeptidyl peptidase-4 (DPP-4) inhibitor revealed anabolic action on skeletal muscle [3, 4], and a recent report showed that DPP-4 inhibitor treatment for 1 year prevented age-associated decline of skeletal muscle as evaluated by dual-energy X-ray absorptiometry (DXA) in the patients with type 2 diabetes [5]. We also reported the effect of 24-week treatment with the DPP4 inhibitor, sitagliptin, and the sulfonylurea, glimepiride, on body composition in Japanese overweight and obese patients with type 2 diabetes. Although the degree of $\mathrm{HbAlc}$ reduction was similar between the two groups, a significant reduction in IHL, as determined by proton magnetic resonance spectroscopy $\left({ }^{1} \mathrm{H}-\mathrm{MRCP}\right)$, and in fat mass (FM), as determined by DXA, were observed in the sitagliptin group but not in the glimepiride group [6]. As excess of body fat is a risk factor for insulin resistance [7], this reduction may lead to improvement of insulin sensitivity, which in turn may contribute to sitagliptininduced amelioration of glycemic control.

Skeletal muscle is a major site of insulin-mediated glucose disposal, and a decrease of muscle mass is inversely associated with insulin resistance [8]. Using the hyperinsulinemic-euglycemic glucose clamp technique [9], muscle and muscle/fat ratio have been reported to be positively correlated with insulin sensitivity; therefore, it may be interesting to evaluate whether 
sitagliptin influenced these factors. In our previous report, we showed no significant change in lean body mass (LBM) in both groups [6]. DXA separates the whole body into three major components, bone mass (BM), FM and fat and bone-free mass (FBFM), and measures the weight of each component. LBM means BM plus FBFM, and thus, it is desirable to use not only LBM but FBFM for strict evaluation of muscle mass. Taken together, in this post-hoc analysis, we investigated whether sitagliptin treatment for 24 weeks influenced FBFM and the FBFM/FM ratio.

\section{Materials and Methods}

We analyzed the data from 20 patients with type 2 diabetes with a body mass index $(\mathrm{BMI}) \geq 25 \mathrm{~kg} / \mathrm{m}^{2}$ or fatty liver enrolled in a prospective, 24-week, single-center, open-label comparative study conducted at the outpatient clinic of St. Marianna University Hospital (Kawasaki, Japan) between June 2011 and January 2014. The subjects were assigned to treatment of sitagliptin $(25 \mathrm{mg}$ titrated to $50 \mathrm{mg})$ or glimepiride $(0.5 \mathrm{mg}$ titrated to $1.0 \mathrm{mg}$ ), and the primary objective was to evaluate the effect on FM and IHL. The second objective was to investigate the effect on glycemic control and adipokine (leptin and high molecular weight of adiponectin) secretion. We selected glimepiride as an active comparator to assess the influence on the plasma glucose level. The results of the study have been published previously [6]. The protocol for this research was approved by the Ethics Committee of St. Marianna University School of Medicine and it conforms to the provisions of the Declaration of Helsinki, and informed consent was obtained from all subjects. This study was registered at the University Hospital Medical Network Clinical Trial Registry (UMIN: 000013356).

\section{Statistical analysis}

Results are expressed as mean \pm standard deviation (SD). All statistical analyses were performed with BellCurve for Excel, Version 3.20 (Social Survey Research Information Co., Ltd, Tokyo, Japan). Comparison of mean data between baseline (week 0) and 12 weeks or 24 weeks in each group was assessed with the paired $t$-test, and comparison of mean change of the data from baseline to 24 weeks between the two groups was assessed using the $t$-test. In all analyses, a probability of less than 0.05 was considered to indicate statistical significance.

\section{Results}

As shown in Table 1, although the FM, FBFM and FBFM/ FM ratio were not changed at 12 weeks in either group, at 24 weeks the FBFM and FBFM/FM ratio were significantly increased and the FM was significantly decreased in the sitagliptin group but not in the glimepiride group. Mean changes in the FBFM and FM and FBFM/FM ratio from baseline to 24 weeks in the sitagliptin group and the glimepiride group are shown in

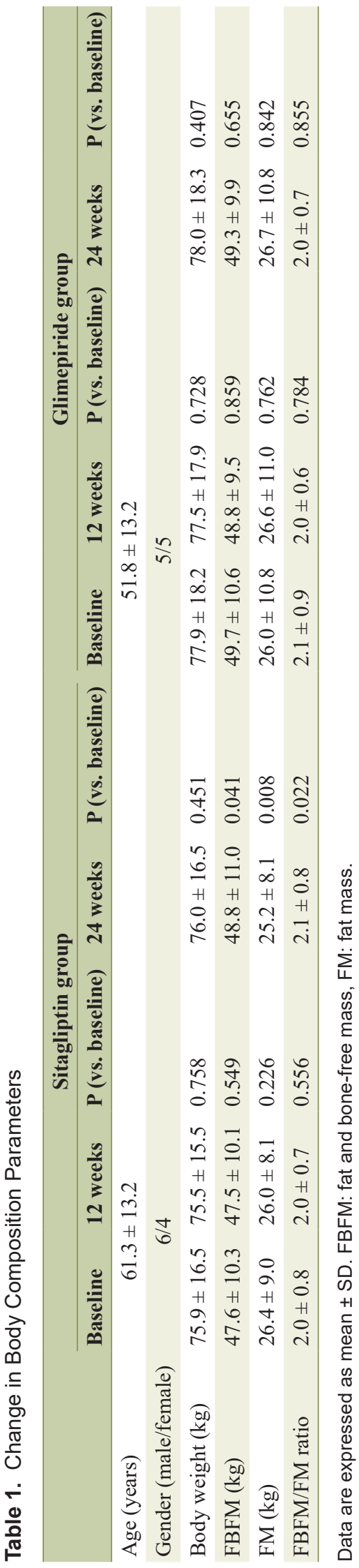



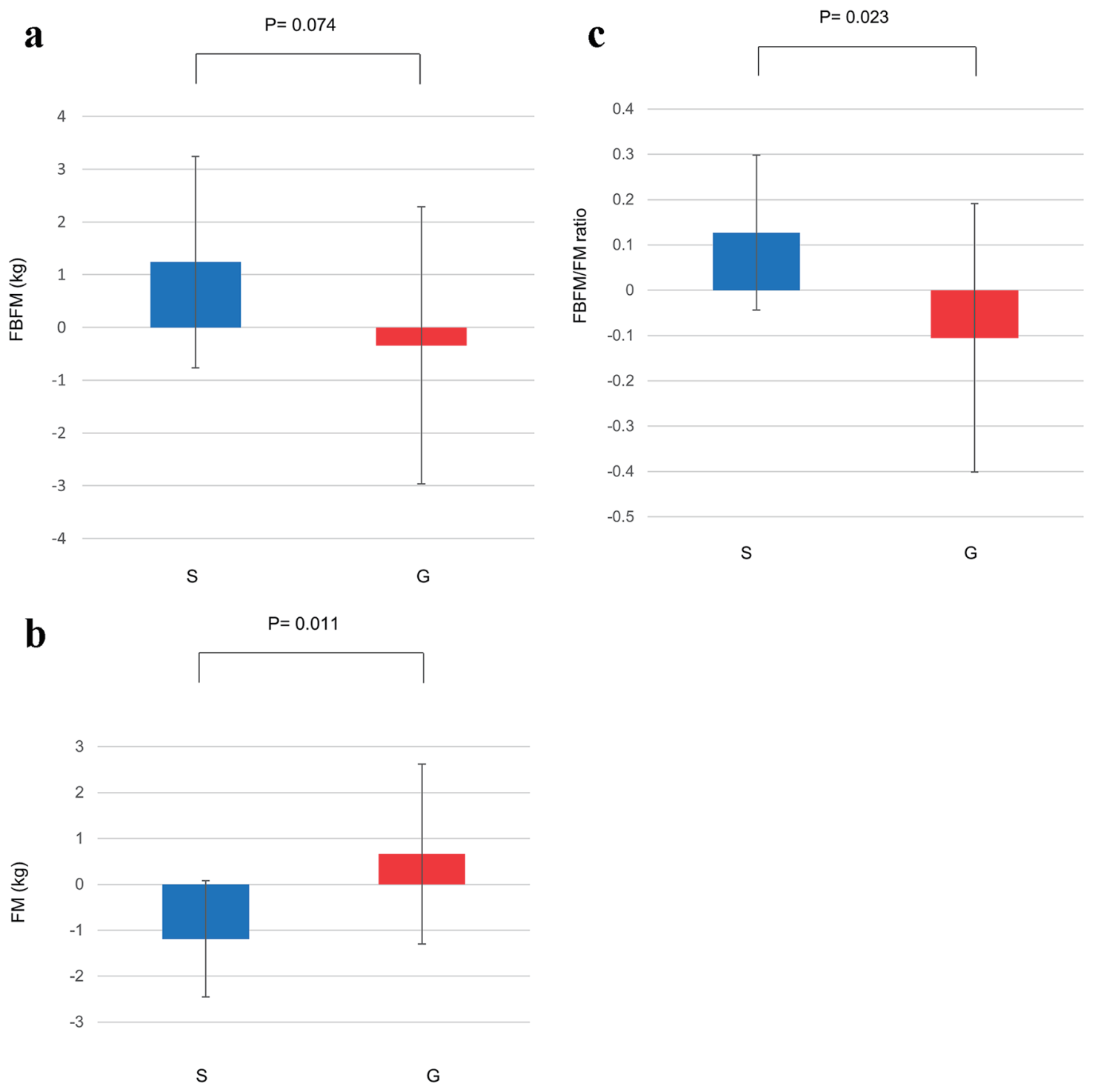

Figure 1. (a) Change in the FBFM from baseline to 24 weeks. (b) Change in the FM from baseline to 24 weeks. (c) Change in the FBFM/FM ratio from baseline to 24 weeks. Data are shown as mean \pm SD. FBFM: fat and bone-free mass; FM: fat mass; G: glimepiride group; S: sitagliptin group; SD: standard deviation.

Figure 1 (FBFM: $1.24 \pm 2.01$ vs. $-0.34 \pm 2.63, \mathrm{P}=0.074$; FM: $-1.19 \pm 1.27$ vs. $0.66 \pm 1.96, \mathrm{P}<0.05 ; \mathrm{FBFM} / \mathrm{FM}$ ratio: $0.13 \pm$ 0.17 vs. $-0.11 \pm 0.30, \mathrm{P}<0.05)$.

\section{Discussion}

Regarding the effect of DPP-4 inhibitors on muscle mass in the patients with type 2 diabetes, a recent study to evaluate the effect of sitagliptin daily $50 \mathrm{mg}$ treatment for 12 weeks showed no significant change in upper and lower limb FBFM (as determined with DXA) in Japanese subjects with a BMI $\geq 23 \mathrm{~kg} / \mathrm{m}^{2}$ [10]. Similar result using DXA also showed that neither FBFM nor FM was changed by 12 weeks of daily sitagliptin $100 \mathrm{mg}$ in Dutch Europid prediabetic men with a BMI $25-35 \mathrm{~kg} / \mathrm{m}^{2}$
[11]. These reports did not evaluate muscle/FM ratio and suggested that the 12-week treatment of sitagliptin may be not enough to affect muscle mass. In the present study, both FBFM and FBFM/FM ratio at 12 weeks were not changed from the baseline levels; however, significant change was observed at 24 weeks.

The mechanism of sitagliptin-induced FBFM and FBFM/ FM ratio increase is unclear. In an animal study, sitagliptin increased glucose transporter-4 (GLUT4) expression in skeletal muscle of diabetic rats [12], and MK-0626, a DPP-4 inhibitor, improved mitochondrial biogenesis and exercise capacity in skeletal muscle in mice with recent myocardial infarction [4]. However, it was not clear whether these effects resulted from enhancing GLP-1 action or the inhibition of DPP-4 activity or both. Regarding GLP-1 action, various results of decrease, 
unchanged and increase of muscle in patients with diabetes with treatment of GLP-1 receptor agonists have been reported [13]. GLP-1 receptor agonists potently inhibit appetite by direct action on the hypothalamus [14], and thus, muscle mass may decrease by reduction of energy intake. Furthermore, not only body fat, but also both muscle volume and muscle strength were decreased by dietary energy restriction in the study of healthy obesity subjects [15]. Thus, when we evaluate the effect of a GLP-1 receptor agonist on body composition, it should be noted that the results may be induced by the direct action of GLP-1 on muscle and fat tissue as well as central action leading to the attenuation of appetite and reduction in energy intake. With respect to DPP-4 action on muscle, soluble DPP-4 was reported to impair muscle insulin signaling in subjects with metabolic syndrome; however, the exact action of DPP-4 on muscle remains unclear [16]. Taken together, further investigation is required to explain the mechanism underlining the effect of DPP-4 inhibitor on muscle tissue.

Examining our previous study and the present post-hoc analysis, the increase of the FBFM/FM ratio by sitagliptin treatment for 24 weeks may result from the decrease of FM and increase of FBFM. Recently, the muscle/fat ratio has been thought as an important marker of insulin sensitivity. Kim et al analyzed 14,807 subjects in the Korean National Health and Nutrition Examination Survey (KNHANES) with DXA data and divided the subjects into four groups by median value of muscle and FM (high muscle/low fat, high muscle/high fat, low muscle/high fat and low muscle/low fat). The group of high muscle/low fat showed a much lower HOMA-R, a marker of insulin resistance, than the other groups, and the group of high muscle/high fat was significantly associated with the prevalence of metabolic syndrome [17]. These data suggest that the protective association of high muscle with insulin resistance and metabolic syndrome may be canceled by high FM. Kurinami et al examined the correlation of parameters of body composition, as measured by bioimpedance analysis, with insulin sensitivity using a hyperinsulinemic-euglycemic clamp study [9], and they showed a significant correlation of muscle/fat ratio but not muscle mass or FM alone with insulin sensitivity index in untreated patients with diabetes. Thus, a small but significant increase of FBFM/FM ratio may be a beneficial effect of sitagliptin treatment and may in part contribute to the improvement of insulin sensitivity.

In conclusion, 24-week treatment with sitagliptin demonstrated not only a decrease in body fat and liver fat but also an increase in muscle and the muscle/fat ratio. All these changes may partly explain the mechanism underlining sitagliptin-induced improvement of glycemic control.

\section{Acknowledgments}

None to declare.

\section{Financial Disclosure}

None to declare.

\section{Conflict of Interest}

The authors declare no conflict of interest.

\section{Informed Consent}

Written informed consent was obtained from all patients.

\section{Author Contributions}

SI designed the study, participated in data collection, performed statistical analysis and wrote the manuscript. HF, HK and NN participated in data collection and edited the manuscript. YN and YT designed the study and edited the manuscript. All authors have approved the final version to be published.

\section{Data Availability}

The data supporting the findings of this study are available from the corresponding author upon reasonable request.

\section{References}

1. Ohta A, Kato H, Ishii S, Sasaki Y, Nakamura Y, Nakagawa T, Nagai Y, et al. Ipragliflozin, a sodium glucose cotransporter 2 inhibitor, reduces intrahepatic lipid content and abdominal visceral fat volume in patients with type 2 diabetes. Expert Opin Pharmacother. 2017;18(14):14331438.

2. Ishii S, Nagai Y, Sada Y, Fukuda H, Nakamura Y, Matsuba R, Nakagawa T, et al. Liraglutide Reduces Visceral and Intrahepatic Fat Without Significant Loss of Muscle Mass in Obese Patients With Type 2 Diabetes: A Prospective Case Series. J Clin Med Res. 2019;11(3):219224.

3. Hong Y, Lee JH, Jeong KW, Choi CS, Jun HS. Amelioration of muscle wasting by glucagon-like peptide-1 receptor agonist in muscle atrophy. J Cachexia Sarcopenia Muscle. 2019;10(4):903-918.

4. Takada S, Masaki Y, Kinugawa S, Matsumoto J, Furihata T, Mizushima W, Kadoguchi T, et al. Dipeptidyl peptidase-4 inhibitor improved exercise capacity and mitochondrial biogenesis in mice with heart failure via activation of glucagon-like peptide-1 receptor signalling. Cardiovasc Res. 2016;111(4):338-347.

5. Bouchi R, Fukuda T, Takeuchi T, Nakano Y, Murakami M, Minami I, Izumiyama H, et al. Dipeptidyl peptidase 4 inhibitors attenuates the decline of skeletal muscle mass in patients with type 2 diabetes. Diabetes Metab Res Rev. 2018;34(2):e2957.

6. Kato H, Nagai Y, Ohta A, Tenjin A, Nakamura Y, Tsukiyama H, Sasaki Y, et al. Effect of sitagliptin on intrahepatic lipid content and body fat in patients with type 2 diabetes. Diabetes Res Clin Pract. 2015;109(1):199-205. 
7. Matsubara F, Nagai Y, Tsukiyama H, Shimizu H, Yamanouchi E, Iwamoto T, Sada Y, et al. Proposed cut-off value of the intrahepatic lipid content for metabolically normal persons assessed by proton magnetic resonance spectroscopy in a Japanese population. Diabetes Res Clin Pract. 2016;119:75-82.

8. Moon SS. Low skeletal muscle mass is associated with insulin resistance, diabetes, and metabolic syndrome in the Korean population: the Korea National Health and Nutrition Examination Survey (KNHANES) 2009-2010. Endocr J. 2014;61(1):61-70.

9. Kurinami N, Sugiyama S, Yoshida A, Hieshima K, Miyamoto F, Kajiwara K, Jinnouchi T, et al. Correlation of body muscle/fat ratio with insulin sensitivity using hyperinsulinemic-euglycemic clamp in treatment-naive type 2 diabetes mellitus. Diabetes Res Clin Pract. 2016;120:6572.

10. Tsurutani Y, Nakai K, Inoue K, Azuma K, Mukai S, Maruyama S, lizuka T, et al. Comparative study of the effects of ipragliflozin and sitagliptin on multiple metabolic variables in Japanese patients with type 2 diabetes: A multicentre, randomized, prospective, open-label, active-controlled study. Diabetes Obes Metab. 2018;20(11):26752679.

11. Nahon KJ, Doornink F, Straat ME, Botani K, MartinezTellez B, Abreu-Vieira G, van Klinken JB, et al. Effect of sitagliptin on energy metabolism and brown adi- pose tissue in overweight individuals with prediabetes: a randomised placebo-controlled trial. Diabetologia. 2018;61(11):2386-2397.

12. Hu H, Xu M, Qi R, Wang Y, Wang C, Liu J, Luo L, et al. Sitagliptin downregulates retinol-binding protein 4 and upregulates glucose transporter type 4 expression in a type 2 diabetes mellitus rat model. Int J Clin Exp Med. 2015;8(10):17902-17911.

13. Sargeant JA, Henson J, King JA, Yates T, Khunti K, Davies MJ. A review of the effects of glucagon-like peptide-1 receptor agonists and sodium-glucose cotransporter 2 inhibitors on lean body mass in humans. Endocrinol Metab (Seoul). 2019;34(3):247-262.

14. Drucker DJ. Mechanisms of Action and Therapeutic Application of Glucagon-like Peptide-1. Cell Metab. 2018;27(4):740-756.

15. Kim B, Tsujimoto T, So R, Zhao X, Oh S, Tanaka K. Changes in muscle strength after diet-induced weight reduction in adult men with obesity: a prospective study. Diabetes Metab Syndr Obes. 2017;10:187-194.

16. Kalaitzoglou E, Fowlkes JL, Popescu I, Thrailkill KM. Diabetes pharmacotherapy and effects on the musculoskeletal system. Diabetes Metab Res Rev. 2019;35(2):e3100.

17. Kim K, Park SM. Association of muscle mass and fat mass with insulin resistance and the prevalence of metabolic syndrome in Korean adults: a cross-sectional study. Sci Rep. 2018;8(1):2703. 